\title{
DEFINITIONS AND PROPERTIES OF MONOTONE FUNCTIONS ${ }^{1}$
}

\author{
G. BALEY PRICE
}

1. Introduction. We shall consider functions which are monotone in the following sense: $x(t)$ is monotone if and only if $x(t)$ is between $x\left(t_{1}\right)$ and $x\left(t_{2}\right)$ whenever $t$ is between $t_{1}$ and $t_{2}$. This definition has content only after betweenness has been defined in the domain and range spaces. It is our purpose to consider several definitions of betweenness and the properties of the corresponding monotone functions.

2. Order-monotone functions. In this section we shall consider functions $x(t)$ defined on an interval of real numbers with values in a linear partially ordered space $X$ or a partially ordered topological group $X$ in the sense of Kantorovitch ${ }^{2}[1]$. We shall say that $x(t)$ is order-monotone if it is monotone according to the definition in the introduction with betweenness defined as follows: $t$ is between $t_{1}, t_{2}$ if and only if $t_{1} \leqq t \leqq t_{2} ; x(t)$ is between $x\left(t_{1}\right), x\left(t_{2}\right)$ if and only if $x\left(t_{1}\right) \leqq x(t) \leqq x\left(t_{2}\right)$. Throughout the remainder of this section, $x(t)$ is assumed to be order-monotone unless there is a statement to the contrary.

If $t_{1}<t_{2}<\cdots$ is a sequence with $t_{0}$ as a limit, it can be shown that $\lim x\left(t_{n}\right)$ exists or is infinite; similarly for a monotone decreasing sequence, $t_{n}^{\prime} \rightarrow t_{0}$. If $\lim x\left(t_{n}\right)=\lim x\left(t_{n}^{\prime}\right)=x\left(t_{0}\right)$, we say that $x(t)$ is continuous at $t_{0}$; otherwise, $x(t)$ is discontinuous there. If $\lim x\left(t_{n}\right)$, $\lim x\left(t_{n}^{\prime}\right)$ both exist but are unequal, we say that $x(t)$ has a jump equal to their difference. It follows from a theorem of Kantorovitch $[1$, p. 130] that when $x(t)$ is order-monotone on a closed interval, the

1 Presented to the Society, September 6, 1938.

${ }^{2}$ Let $X$ be a class of elements $x$ which form an additive abelian group. Furthermore, let there be a relation $>$ defined so that for some of the elements $x \varepsilon X$ the relation $x>0$ holds. We assume that this relation satisfies the following postulates: I. The relation $x>0$ excludes the relation $x=0$. II. If $x_{1}>0$ and $x_{2}>0$, then $x_{1}+x_{2}>0$. III. To each element $x \varepsilon X$ there corresponds at least one element $x_{1} \varepsilon X$ such that $x_{1} \geqq 0$ and $x_{1}-x \geqq 0$. IV. If $x>0$ and $\lambda>0$ is a real number, then $\lambda x>0$. V. For every set $E$ bounded above there exists a least upper bound sup $E$.

If I, II, III, $V$ are satisfied in $X$, it is called a partially ordered topological group. If in addition IV is satisfied in $X$, it is called a linear partially ordered space.

If $x_{2}-x_{1}>0$, we say $x_{2}>x_{1}$. In a partially ordered space it is possible to define an absolute value $|x|$ of $x$; the absolute value of $x$ is an element in the space and has the formal properties of the absolute value of a real number. For the definition of $|x|$, the definitions and properties of limits, and other results, the reader is referred to the paper of Kantorovitch. 
number of points of discontinuity at which the jump is equal to or greater than a fixed element in $X$ is finite. ${ }^{3}$ Furthermore, if $x_{1}(t)$, $x_{2}(t)$ are order-monotone in the same sense, their sum $x_{1}(t)+x_{2}(t)$ is order-monotone in that sense also.

Let $x(t)$ be any function from $a \leqq t \leqq b$ to a partially ordered topological group $X ; x(t)$ is not assumed to be order-monotone. Subdivide $a \leqq t \leqq b$ by points $t_{i}$ such that $a=t_{0}<t_{1}<\cdots<t_{n}=b$, and form the sums $\sum_{i=0}^{n-1}\left|x\left(t_{i+1}\right)-x\left(t_{i}\right)\right|$. If the set of such sums formed for all subdivisions of $a \leqq t \leqq b$ has an upper bound (a finite element of $X$, and not an improper upper bound of the entire space), we say $x(t)$ has bounded variation. Furthermore,

$$
\sup \left\{\sum_{i=0}^{n-1}\left|x\left(t_{i+1}\right)-x\left(t_{i}\right)\right|\right\}
$$

is called the total variation of $x(t)$ on $a \leqq t \leqq b$ and is denoted by $V_{a}^{b}[x(t)]$. If $x(t)$ is order-monotone, it can be shown that it has total variation equal to $|x(b)-x(a)|$. Let $x(t)$ be a function of bounded variation from $a \leqq t \leqq b$ to a partially ordered topological group $X$; it can be expressed as the difference of two order-monotone increasing functions which are bounded and greater than zero for each value of $t$. In particular, we can show that

$$
x(t)=\left\{V_{a}^{t}[x(s)]+x_{0}\right\}-\left\{V_{a}^{t}[x(s)]+x_{0}-x(t)\right\},
$$

where $x_{0}$ is a properly chosen constant, is a representation of the form stated. Furthermore, it is easily shown that the linear extension of the set of order-monotone functions $x(t)$ from $a \leqq t \leqq b$ to a linear partially ordered space $X$ is the class of functions of bounded variation.

Finally, let $x_{1}(t), x_{2}(t), \cdots$ be a sequence of order-monotone functions defined on $a \leqq t \leqq b$ such that $\lim x_{n}(t)$ exists for each $t$ and equals $x(t)$. Then $x(t)$ likewise is order-monotone.

3. Metric-monotone functions. We shall now consider functions $x(t)$ defined on an interval of real numbers with values in a complete metric space $X$ with elements $x_{1}, x_{2}$ and distance function $\rho\left(x_{1}, x_{2}\right)$. We shall say that $x(t)$ is metric-monotone if it is monotone according to the definition in the introduction with betweenness defined as follows: $t$ is between $t_{1}, t_{2}$ if and only if $t_{1} \leqq t \leqq t_{2} ; x(t)$ is between $x\left(t_{1}\right)$,

${ }^{3}$ Let $n x$ denote the sum of $n$ elements $x$; Kantorovitch has shown that the set of elements $n x,(n=1,2, \cdots)$, is unbounded (see postulate V). If $x(t)$ is order-monotone increasing, for example, on $a \leqq t \leqq b$ and has $n$ points of discontinuity at which the jump exceeds $x$, then $x(b)-x(a)>n x$, from which the result stated follows. 
$x\left(t_{2}\right)$ if and only if $\rho\left[x\left(t_{1}\right), x\left(t_{2}\right)\right]=\rho\left[x\left(t_{1}\right), x(t)\right]+\rho\left[x(t), x\left(t_{2}\right)\right]$ (compare a definition of betweenness given by Menger [2, pp. 77-81]).

If $x(t)$ is metric-monotone on an interval, its discontinuities are either simple discontinuities or infinities, and the latter can occur only at the ends of the interval of definition. Let the jump at a simple discontinuity be defined as the distance between the two limits. If the interval of definition of a metric-monotone function is closed, the number of discontinuitities at which the jump exceeds a given constant is finite, and the set of all discontinuities is denumerable. The variation of a function $x(t)$ with values in a metric space is defined as a number in the usual way. The decomposition of a function $x(t)$ of bounded variation with values in a normed vector space into a sum of metric-monotone functions is lacking; it appears that this decomposition depends essentially on relations of order. If $x_{1}(t), x_{2}(t), \cdots$ is a sequence of metric-monotone functions defined on $a \leqq t \leqq b$, and if $\lim x_{n}(t)$ exists for each $t$ and equals $x(t)$, then $x(t)$ is also metric monotone.

4. Sphere-monotone functions. In this section we shall consider functions $x(t)$ defined on an interval with values in a metric space $X$. We shall say that $x(t)$ is sphere-monotone if and only if it is monotone according to the definition in the introduction with betweenness defined as follows: $t$ is between $t_{1}, t_{2}$ if and only if $t_{1} \leqq t \leqq t_{2} ; x(t)$ is between $x\left(t_{1}\right), x\left(t_{2}\right)$ if and only if every open sphere which contains $x\left(t_{1}\right), x\left(t_{2}\right)$ also contains $x(t)$.

The discontinuities of a sphere-monotone function are of certain simple types only. Let $t_{1}<t_{2}<\cdots$ be a sequence of numbers on the interval of definition of $x(t)$, and let $t_{n} \rightarrow t_{0}$. Then either $x\left(t_{n}\right)$ tends to a limit and $x(t)$ has a limit at $t_{0}$ on the left equal to it, or the set $\left\{x\left(t_{n}\right)\right\}$ has no point of accumulation in $X$. If $t_{0}$ is an interior point of the interval of definition of $x(t)$, similar results hold at $t_{0}$ on the right. Thus besides simple discontinuities and infinities, a spheremonotone function has a third type of discontinuity that may be described as wandering. As in all previous cases, an infinity can occur only at an end point of an open interval on which $x(t)$ is defined. There are no wandering discontinuities at least when the set of values of $x(t)$ is compact.

In all previous cases, a monotone function has been a function of bounded variation; a sphere-monotone function, however, need not have bounded variation (see an example given by Graves [3, p. 166]). If $x_{1}(t), x_{2}(t), \cdots$ is a sequence of sphere-monotone functions, and if $\lim x_{n}(t)$ exists for each $t$ and equals $x(t)$, then $x(t)$ is also spheremonotone. 
A function $x(t)$ defined on an interval with values in a metric space $X$ is said to be measurable if and only if for every open sphere $S$ in $X$ the set $E_{t}[x(t) \varepsilon S]$ is measurable. Every sphere-monotone function $x(t)$ is measurable in this sense. Bochner [4, pp. 263-265] has also given a definition of measurable function. A necessary and sufficient condition that $x(t)$ be measurable in the sense of Bochner is that it be measurable, and that the set of values of $x(t)$, omitting at most those corresponding to $t$ on a set of measure zero, be separable. The function in the example of the last paragraph (Graves [3, p. 166]) is sphere-monotone and hence measurable, but since the distance between each two of its values is one, it does not satisfy the condition for Bochner measurability. There are thus measurable functions which are not measurable in the sense of Bochner.

5. Other monotone functions. It is possible to define still other types of monotone functions; all that is required is that betweenness be defined in both the domain and range spaces of $x(t)$. In particular, let $x(t)$ be a function defined on an "interval" $a \leqq t \leqq b$ of a partially ordered topological group with values in a space of the same kind. The number of points of discontinuity at which the jump exceeds a given constant may not be finite; with this exception, the results given in $\$ 2$ hold for this monotone function also. It should be observed that the Arzelà real-valued monotone functions of several variables are special cases of these monotone functions.

In a linear space, the following definition of betweenness is a natural one: $x$ is between $x_{1}, x_{2}$ if and only if $x=\theta x_{1}+(1-\theta) x_{2}$, where $\theta$ is a real number such that $0 \leqq \theta \leqq 1$. Corresponding to this definition of betweenness we have linear-monotone functions; apparently they have not been studied previously. An account of them will be given elsewhere.

\section{REFERENCES}

1. L. V. Kantorovitch, Lineare halbgeordnete Räume, Recueil Mathématique, new series, vol. 2 (1937), pp. 121-165.

2. K. Menger, Untersuchungen iber allgemeine Metrik, Mathematische Annalen, vol. 100 (1928), pp. 75-163.

3. L. M. Graves, Riemann integration and Taylor's theorem in general analysis, Transactions of this Society, vol. 29 (1927), pp. 163-177.

4. S. Bochner, Integration von Funktionen, deren Werte die Elemente eines Vektorraumes sind, Fundamenta Mathematicae, vol. 20 (1933), pp. 262-276.

UNIVERSITY OF KANSAS 